in $56 \%$ of handovers - including rota gaps, sickness and cancelled locums.

The non-clinical rota coordinator now feels empowered to raise key staffing issues and the staff have commented that it has provided a good snapshot of all the clinical areas. Data from the next 20-week period and a further qualitative survey on how it has improved staff's situation awareness are being compiled.

Conclusion The introduction of a handover checklist can provide a structure and give staff an overview of the department's clinical risk, therefore it can improve patient safety. Whilst it is easy to obtain numerical data on the length of handovers and whether key issues were discussed, further work is required to delineate secondary outcomes - staff's situational awareness/bandwidth and impact on departmental teaching.

\section{G137(P) FOOD MATTERS: A MULTIDISCIPLINARY INITIATIVE TO RAISE FOOD ALLERGY AWARENESS AND SAFETY ON A PAEDIATRIC WARD}

I Gerry, A Freedman, S Green, K Gudka, L Gibb, H Dejesus, E Lee, M Gandhi. Paediatrics, Royal Free Hospital, London, UK

\subsection{6/archdischild-2020-rcpch.110}

Background Increasing numbers of children who attend hospital have co-existing food allergies, whilst the incidence of children with food allergies worldwide continues to rise. Anxiety and confusion exist amongst these patients, carers and healthcare staff about food allergy awareness. There is currently no national universal training programme in place for healthcare professionals and other staff members.

Objectives

- To improve the safety of children with food allergies attending the paediatric ward

- To develop a structured training tool and implement a universal training programme for all healthcare professionals and staff.

Methods We undertook a quality improvement project to develop a structured teaching tool to train ward staff at all levels on food allergens and labelling, identification of children with food allergies and acute allergy management. A multidisciplinary team involving doctors, nurses, dietician, housekeepers, teachers and physician assistants worked on this initiative. Teaching was facilitated using microteach face to face sessions to ensure maximum staff uptake on the 'shop floor'. A pre and post-teaching questionnaire was undertaken to evaluate the efficacy of the teaching sessions.

Results 23 members of staff were trained including nurses, doctors, housekeepers, ward teachers, caterers and dieticians. During training, we identified that 22/23 (95\%) of staff members were willing to guess what food allergens were in a processed food based on prior knowledge without checking the label. Prior to training 9/23 staff members had difficulty understanding food allergen labelling and laws. We were able to address common misconceptions during training using practical examples. During training staff were taught to identify symptoms of food allergy and how to use an adrenaline autoinjector. After training 12/23 (52\%) said that they felt much more knowledgeable about food allergies. 22/23 (95\%) staff members trained felt confident in when and how to use an adrenaline autoinjector compared to $13 / 23$ prior to training.

Conclusions Implementing a universal food allergy awareness programme for all staff on the ward where food is eaten and enjoyed is an important step towards safeguarding children on the ward with food allergies.

\section{G138(P) UTILISING SIMPLE TECHNOLOGY TO IMPROVE MANAGEMENT OF TYPE 1 DIABETES PATIENTS}

${ }^{1} \mathrm{D}$ Dodia, ${ }^{2} \mathrm{~S}$ Wassouf, ${ }^{2} \mathrm{M}$ Watson. 'Medicine, Imperial College London, London, UK; ${ }^{2}$ Paediatric Diabetes, St. Mary's Hospital, London, UK

\subsection{6/archdischild-2020-rcpch.111}

Background and Aims The Paediatric Diabetes team of an inner-city hospital use Microsoft Excel to record Haemoglobin A1c (HbA1c) measurements from 116 patients. HbA1c levels inform the clinician of long-term glycaemic control and allow adaptation of the patient management plan.

Manual editing spreadsheets is an arduous process with a risk of human error. IT literacy of staff, training times, and costs limit the use of technological advances. Microsoft Excel - a cheap, simple, and easily accessible program - empowers teams to create dynamic spreadsheets. We aimed to increase the time efficiency of the Paediatric Diabetes team by improving their spreadsheet.

Methods The original HbA1c spreadsheet was assessed using a questionnaire and team discussion. The spreadsheet was amended based on team requirements and suggestions. Data grouping was used to simplify the spreadsheet. Conditional formatting highlighted missing data and appointments, upcoming appointments, and colour coded $\mathrm{HbA} 1 \mathrm{c}$ values within certain ranges. Summaries were evaluated using 'COUNTIF' and 'AVERAGE' formulae. Four hours were spent by a medical student implementing these changes.

Results Feedback from the team was extremely positive, with one person commenting on the intuitiveness and easeof-use. Post-implementation questionnaire responses noted that the spreadsheet had higher accuracy and improved layout. $100 \%$ of participants reported the new spreadsheet was easier to use. The time taken for data input by healthcare professionals reduced by $77.7 \%$ (from 45 to 10 minutes per month).

Conclusions Better data presentation highlighted patients with higher HbA1c levels to healthcare professionals, therefore allowing further support to be provided and thus improving patient care. Automation of formatting and spreadsheet summarising reduced time spent on the document and enabled healthcare professionals to arrange appointments with ease. An explanation of any complex formulae used was provided, to ensure that the document remains usable to those without high IT proficiency.

This idea could be adapted to various teams, especially those managing chronic conditions. Clinicians could consider investing time in straightforward programs like these, as they can be effective at a lower cost than new technological advances.

\section{G139(P) ABSTRACT WITHDRAWN}

